$H$. luzonensis are both descendants of $H$. erectus populations that evolved separately on their respective islands for hundreds of thousands of years, if not more ${ }^{13,14}$. However, explaining the many similarities that $H$. floresiensis and $H$. luzonensis share with early Homo species and australopiths as independently acquired reversals to a more ancestral-like hominin anatomy, owing to evolution in isolated island settings, seems like a stretch of coincidence too far ${ }^{15}$.

Given the rich history of the Out of Africa I paradigm, unsurprisingly, $H$. erectus has been the centre of attention in ideas about early hominin evolution and dispersals in Asia. Nevertheless, it is worth considering how different these ideas might be if, in the 1890s,
$H$. floresiensis or H. luzonensis had been discovered rather than $H$. erectus. Because $H$. luzonensis provides the first glimpse of a second hominin species living on a distant island at a time when $H$. sapiens populations from Africa were beginning to spread across the world, one thing can be said for certain - our picture of hominin evolution in Asia during the Pleistocene just got even messier, more complicated and a whole lot more interesting.

Matthew W. Tocheri is in the Department of Anthropology, Lakehead University, Thunder Bay, Ontario P7B 5E1, Canada.

e-mail:mtocheri@lakeheadu.ca

1. Détroit, F. et al. Nature 568, 181-186 (2019).
2. Ferring, R. et al. Proc. Natl Acad. Sci. USA 108, 10432-10436 (2011).

3. Fleagle, J. G. et al. (eds) Out of Africa I: the First Hominin Colonization of Eurasia (Springer, 2010).

4. Hershkovitz, I. et al. Science 359, 456-459 (2018).

5. Dennell, R. \& Roebroeks, W. Nature 438, 10991104 (2005)

6. Zhu, Z. et al. Nature 559, 608-612 (2018).

7. Brown, P. et al. Nature 431, 1055-1061 (2004).

8. Mijares, A. S. et al. J. Hum. Evol. 59, 123-132 (2010).

9. Kivell, T. L. et al. Nature Commun. 6, 8431 (2015) 10.Morwood, M. J. et al. Nature 437, 1012-1017 (2005).

11.van den Bergh, G. D. et al. Nature 534, 245-248 (2016).

12.Argue, D., Groves, C. P., Lee, M. S. Y. \& Jungers, W. L. J. Hum. Evol. 107, 107-133 (2017).

13. Brumm, A. et al. Nature 441, 624-628 (2006).

14.Ingicco, T. et al. Nature 557, 233-237 (2018)

15.Morwood, M. J. \& Jungers, W. L. J. Hum. Evol. 57, 640-648 (2009).

\title{
Correlations detected in a quantum vacuum
}

A vacuum as described by quantum mechanics is perhaps the most fundamental but mysterious state in physics. The discovery of correlations between electricfield fluctuations in such a vacuum represents a major advance. SEE LETTER P.202

\section{ANDREY S. MOSKALENKO \& TIMOTHY C. RALPH}

A surprising result in quantum mechanics is that a vacuum is not empty. Particles can appear out of nothing for very short periods of time. This phenomenon can be understood as a consequence of the energytime uncertainty principle, whereby restriction of a measurement to an extremely short time interval leads to large fluctuations in energy in the interval. Although indirect effects of these 'virtual' particles are well studied, it is only by probing a vacuum on very short timescales that the particles become 'real' and can be directly observed $^{1}$. But do these particles appear completely randomly, or are they correlated in space and time? On page 202, Benea-Chelmus et al. ${ }^{2}$ provide an answer to this question by finding evidence for correlations between fluctuations in the electric field of a vacuum.

One way to measure correlations in fields is through interference, such as in the doubleslit experiment of British physicist Thomas Young $^{3}$. In this experiment, light waves pass through two slits and interfere with each other to produce an interference pattern on a screen. This simple, but profound, experiment was originally developed to probe wave effects and was later used to illuminate the duality between particles and waves in quantum physics. In the past, variations of the double-slit experiment have been realized for photons, electrons, atoms and large molecules ${ }^{4}$. Current attempts are even looking for multipath interferences for biological objects, such as viruses ${ }^{5}$.

A comparably counter-intuitive enterprise is to search for interferences between separated parts of a vacuum. Benea-Chelmus and colleagues devoted their experimental study to exactly this task. For a simple, conceptual

physical explanation of their work, consider a version of the double-slit experiment that is based on an instrument called a MachZehnder interferometer ${ }^{6}$ (Fig. 1a). Moreover, let us limit the discussion to temporal correlations and consider the case in which thermal radiation is incident on the interferometer.

In this set-up, the radiation is divided into two equal parts by a beam splitter. The two parts propagate in their own 'arms' of the interferometer before passing through a second beam splitter and being collected by two detectors. In one of the arms, there is a device called a delay line, which introduces a variable time delay in the propagation of one part with respect to the other. The correlation properties of the radiation can be determined from the variation in the intensity measured by either of the detectors as a function of the time delay. A perfect interference pattern (one that is at its maximum visibility) is observed if the intensity oscillates between a certain maximum

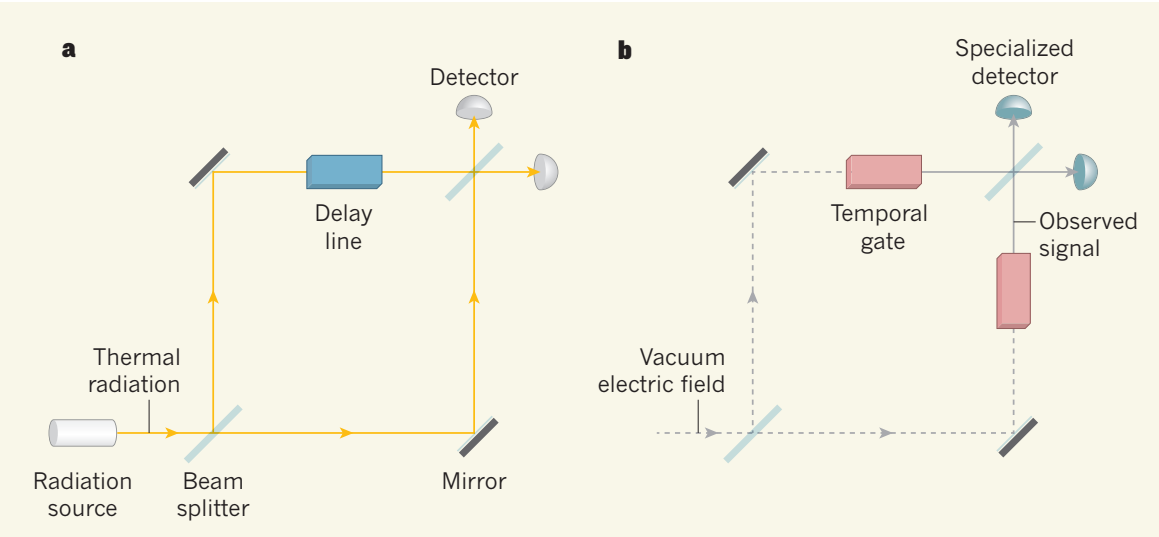

Figure 1 | Standard and modified Mach-Zehnder interferometers. a, In a Mach-Zehnder interferometer, thermal radiation from a radiation source is split into two parts by a beam splitter. Each part is directed by a mirror through a second beam splitter and into a detector. A device called a delay line introduces a time delay in the propagation of one part with respect to the other. This set-up can be used to study the correlation properties of the radiation. $\mathbf{b}$, Benea-Chelmus et al. ${ }^{2}$ report an experiment that can be thought of as a modified Mach-Zehnder interferometer. The radiation and its source are replaced by the electric field associated with a vacuum, and the delay line is substituted with two elements called temporal gates that 'observe' electric fields in ultrashort time windows - the two observations differ by a time delay. Specialized detectors analyse the observed signals. The authors used their experiment to measure correlations between electric-field fluctuations in the vacuum. 
value and zero.

Even though thermal radiation is completely chaotic, an almost perfect interference pattern can be seen in such a set-up for small time delays. However, when the time delay becomes larger than a temperature-dependent quantity called the coherence time, the amplitude of the oscillations with respect to the maximum value quickly decays and the minimum value is no longer zero. Benea-Chelmus et al. observed this behaviour in their experiment at about room temperature (300 kelvin) and found that the coherence time agreed with theoretical predictions.

If the temperature is lowered to a few kelvin, radiation in the terahertz frequency range, which is relevant to such experiments, is suppressed. For example, at $4 \mathrm{~K}$, there are effectively no photons that have frequencies larger than $0.2 \mathrm{THz}$. As a result, in the standard set-up, the maximum amplitude of the oscillations drops to zero and there is no evidence of correlations. Remarkably, Benea-Chelmus and colleagues found that they could still observe correlations in their version of the set-up at $4 \mathrm{~K}$. They interpret these signals as a direct signature of correlations in the electric field of the vacuum.

The authors achieved this feat because of a sophisticated detection scheme. Based on a branch of research known as nonlinear optics, the scheme simultaneously provides two key components that can be pictured in terms of a modified Mach-Zehnder interferometer (Fig. 1b). The first is a pair of elements called temporal gates that 'observe' electric fields in ultrashort time windows - the two observations differ by a time delay. In Benea-Chelmus and colleagues' experiment, these gates are implemented by auxiliary ultrashort pulses of near-infrared light that interact with the vacuum in a nonlinear optical crystal. The second is a pair of detectors that are directly sensitive to the electric fields that have passed through the temporal gates and to the correlations in these fields.

Benea-Chelmus et al. note that the maximum amplitude of the oscillations, although non-zero, was so small that up to one trillion individual detection events at each value of the time delay were required

"The authors
found that they
could still observe
correlations in
their version of
the set-up at
4 kelvin."

for the correlations to be discernible from measurement-induced random fluctuations (shot noise). Such a large number of events took about 3 hours at a repetition rate of $80 \mathrm{MHz}$.

An intriguing aspect of the experiment that deserves further investigation is the effect of the detection procedure, including the use of temporal gates, on the measured fields - a phenomenon called quantum back-action ${ }^{7}$.

Previous work has described how photons materialize in a vacuum for hypothetical, short-lived observers that, because of the finite speed of light, can access information only from restricted regions of space-time, called space-time diamonds ${ }^{8}$. By considering multiple observers in such settings, it has been shown that the photons display quantum correlation called entanglement ${ }^{9}$. The field of quantum optics began with simple experiments that probed amplitude and intensity correlations in optical fields, but now witnesses and applies sophisticated entanglement-based protocols in nascent quantum technologies. Perhaps the authors' results are the first steps towards ultrafast quantum optics that will one day observe and control the entanglement that lies hidden in the spacetime vacuum and in non-trivial ground states of interacting light-matter systems.

Andrey S. Moskalenko is in the Department of Physics, Korea Advanced Institute of Science and Technology, Daejeon 34141, South Korea, and in the Department of Physics, University of Konstanz, Germany. Timothy C. Ralph is at the School of Mathematics and Physics, The University of Queensland, St Lucia, Queensland 4072, Australia.

e-mails: moskalenko@kaist.ac.kr; ralph@physics.uq.edu.au

1. Riek, C. et al. Science 350, 420-423 (2015).

2. Benea-Chelmus, I.-C., Settembrini, F. F., Scalari, G. \& Faist, J. Nature 568, 202-206 (2019).

3. Young, T. Phil. T. R. Soc. Lond. 92, 12-48 (1802).

4. Arndt, M. \& Hornberger, K. Nature Phys. 10 271-277 (2014).

5. Ananthaswamy, A. Through Two Doors at Once: The Elegant Experiment That Captures The Enigma of Our Quantum Reality (Dutton, 2018).

6. Grangier, P., Roger, G. \& Aspect, A. Europhys. Lett. 1, 173-179 (1986).

7. Braginsky, V. B. \& Khalili F. Y. Quantum Measurement (Cambridge Univ. Press, 1992).

8. Martinetti, P. \& Rovelli, C. Class. Quant. Grav. 20 4919-4931 (2003).

9. Su, D. \& Ralph, T. C. Phys. Rev. D 93, 044023 (2016).

\section{Genetic paradox explained by nonsense}

\section{Gene mutations that truncate the encoded protein can trigger the expression of} related genes. The discovery of this compensatory response alters our thinking about genetic studies in humans and model organisms. SEE ARTICLE P.193 \& LETTER P.259

\section{MILES F. WILKINSON}

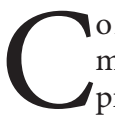
onventional wisdom holds that modifying a gene to make the encoded protein inactive - 'knocking out' the gene - will have more severe effects than merely reducing the gene's expression level. However, there are many cases in which the opposite occurs. In fact, the knockout of a gene sometimes has no discernible impact, whereas the reduction of expression (knockdown) of the same gene causes major defects ${ }^{1}$. Off-target or toxic effects of the reagents used for gene knockdown have sometimes been found to be the culprit ${ }^{2}$, but not always ${ }^{3}$, leaving one to wonder what else could be responsible. El-Brolosy et al. ${ }^{4}$ (page 193) and Ma et al. ${ }^{5}$ (page 259) provide an intriguing solution to this paradox.

The authors identify a molecular mechanism that activates the transcription of genes related to an inactivated gene, thereby compensating for the knockout. The existence of such a genetic compensation response was initially suggested by earlier studies, most notably the discovery ${ }^{3}$ that knockout of the egfl7 gene in zebrafish upregulates the expression of genes that encode proteins related to those encoded by egfl7. This upregulatory response was triggered only by mutation of egfl7, and not by egfl7 knockdown, thereby explaining why knockdown caused biological defects, whereas knockout largely did not. Similar observations have been made for other genes $^{3,6}$, suggesting the existence of a general compensatory mechanism.

How does the compensatory mechanism work? By studying the effects of a variety of mutations in zebrafish embryos, both El-Brolosy et al. and Ma et al. found that the upregulation of compensatory genes is specifically triggered by mutations that generate short nucleotide sequences known as premature termination codons (PTCs). These sequences - also known as nonsense codons - signal the early cessation of the translation of messenger RNAs into proteins. Thus, an apt name for this upregulatory response is nonsense-induced transcriptional compensation (NITC).

The role of PTCs in NITC suggested the involvement of an RNA-degradation pathway called nonsense-mediated RNA decay (NMD), because NMD is also triggered by PTCs ${ }^{7}$. In support of this idea, El-Brolosy and colleagues 